\title{
An Effective Approach to Contention Based Bandwidth Request Mechanism in WiMAX Networks \\ Sonia \\ Satinder Pal
}

Department of Computer Science and Engineering

Indo Global College of Engineering, Abhipur, Mohali

\begin{abstract}
In this paper the IEEE 802.16 standard based Mobile WiMAX (Worldwide Interoperability for Microwave Access) system is investigated for the purpose of Quality of Service provisioning. As a potential solution, scheduling algorithms have been taken into major concern. Within the pool of scheduling algorithms and for the purpose Contention based bandwidth request resolution, the Modified Contention Based BW Resolution algorithm (MCB-BWR-Scheme) is proposed. Supported by the fact that the standard does not emphasize a specific scheduling algorithm for contention period based services, therefore, the choice of the scheduling algorithm for the WiMAX systems is very important. There are several scheduling algorithms for WiMAX in the literature, however, studies show that an efficient, fair and robust scheduling algorithm for WiMAX systems is still an open research area and hence a well thought out algorithm will be of great contribution to the area under investigation. Results from the OPNET Modeler simulation program show that the adjusted algorithm works well with delay and throughput constraints. It is also in consistent with the quality of service demands of the video and voice traffic.
\end{abstract}

General Terms

WiMAX; Backoff Algorithm; Contention Resolution.

Keyword

WiMAX; IEEE 802.16; Modified RED

\section{INTRODUCTION}

\subsection{Worldwide Interoperability Microwave Access (WiMAX)}

Broadband Wireless Access (BWA) has become the easiest way for wireless communication and a solution to rapid requirement of internet connection for data, voice and video service. BWA is a fast and easy alternative of cable networks and Digital Subscriber Line (DSL) technologies. The IEEE working group has designed a new standard based on BWA systems for last mile wireless access named IEEE 802.16 Wireless MAN [8. The IEEE 802.16 architecture is designed to achieve goals like easy deployment, high speed data rate, large spanning area, and large frequency spectrum. The IEEE 802.16 standard provides QoS to all different kinds of application including real time traffic in the form of flow type association with each application.

The above stated advantages of IEEE 802.16 Wireless MAN prepare a platform for this standard to compete with other wireless communication technologies like IEEE 802.11 and its variants. Subsequently the requirement from IEEE 802.16 is to provide QoS for all possible applications in both (uplink and downlink) directions. The IEEE 802.16 is likely to emerge as a dominant technology for cost-competitive ubiquitous broadband wireless access, supporting fixed,
Department of Computer Science and Engineering Indo Global College of Engineering, Abhipur, Mohali

nomadic, portable and fully mobile operations offering integrated voice, video and data services.

The basic IEEE 802.16 architecture [9] consists of one Base Station (BS) and one (or more) Subscriber Stations (SSs). Both BS and SS are stationary while clients connected to SS can be mobile. BS acts as a central entity to transfer all the data from SSs in PMP architecture. Any two (or more) SSs are not allowed to communicate directly. Transmissions take place through two independent channels-Downlink Channel (from BS to SS) and Uplink Channel (from SS to BS). Uplink channel is shared between all the SSs while downlink channel is used only by BS.

The standard defines both Time Division Duplexing (TDD) and Frequency Division Duplexing (FDD) for channel allocation. Both channels are time slotted and composed of frames. The TDD frame composed of downlink and uplink sub frames. The duration of each of these frames can be controlled by BS whenever needed. Downlink channel is broadcast channel. BS broadcast data to all SS on downlink channel. SSs accept only those

- Packets which are destined to it. More details on this architecture can be found in IEEE 802.16 draft [8]. The Figure shows the BS and SS architecture connected to each other through wireless links.

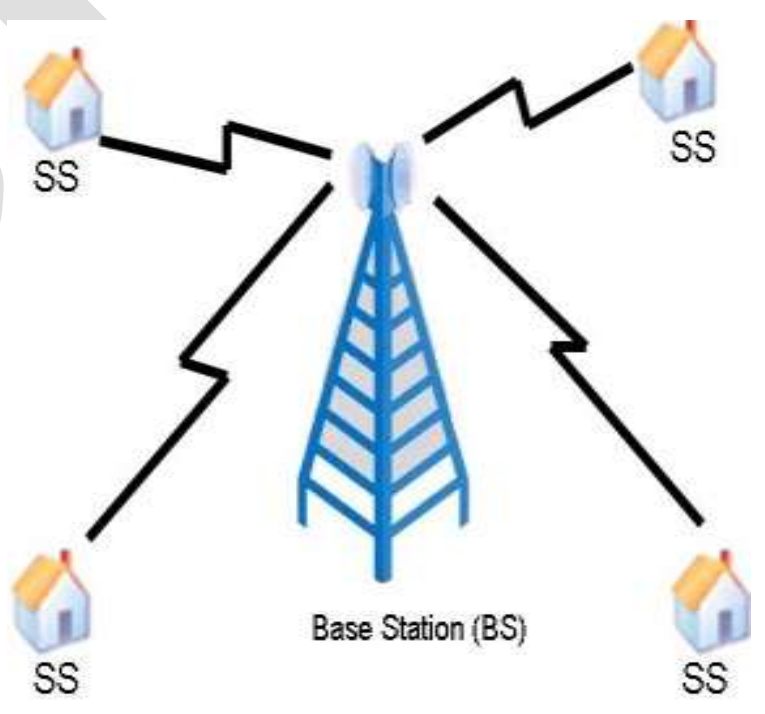

Fig.1 WiMAX Architecture

\section{IEEE 802.16 SERVICE CLASSES}

The IEEE 802.16-2004 standard [3] specifies the provision of four scheduling services:

Table 1.scheduling Services 
Council for Innovative Research www.ijctonline.com ISSN: 2277-3061
International Journal of Computers \& Technology

Volume 3, No. 1, AUG, 2012

\begin{tabular}{|c|l|c|}
\hline Service Class & \multicolumn{1}{|c|}{ Description } & Applications \\
\hline $\begin{array}{c}\text { Unsolicited } \\
\text { Grant Service } \\
\text { (UGS) }\end{array}$ & $\begin{array}{l}\text { For Constant Bit } \\
\text { Rate (CBR) and } \\
\text { delay-dependent } \\
\text { applications }\end{array}$ & \\
\hline $\begin{array}{c}\text { Real-Time } \\
\text { Polling Service } \\
\text { (rtPS) }\end{array}$ & $\begin{array}{l}\text { For Variable Rate } \\
\text { and delay } \\
\text { dependent } \\
\text { applications }\end{array}$ & Streaming video \\
\hline $\begin{array}{c}\text { Extended } \\
\text { Real-Time } \\
\text { Polling Service } \\
\text { (ertPS) }\end{array}$ & $\begin{array}{l}\text { For Variable Rate } \\
\text { and delay } \\
\text { dependent } \\
\text { applications }\end{array}$ & \\
\hline $\begin{array}{c}\text { Best Effort (BE) } \\
\text { Best Effort }\end{array}$ & FTP \\
\hline $\begin{array}{c}\text { Non-real-time } \\
\text { Polling Service } \\
\text { nrtPS) }\end{array}$ & $\begin{array}{l}\text { Variable rate and } \\
\text { non-real time } \\
\text { applications }\end{array}$ & VOIP with silence \\
suppression \\
\hline
\end{tabular}

\section{CONTENTION ACCESS IN IEEE 802.16 MAC}

The MAC layer of IEEE 802.16 specifies the rules for the contention-mode BW request. A contention period is a predetermined number of minislots at the beginning of a UL subframe.

The contention period is divided into an integer number of transmission opportunities (TOs) and is called an information element. Each TO can be used for transmitting only one BW request. If more than one station tries to transmit in the same TO, collision happens. Since it is not practically possible for SSs to sense the UL channel to detect a collision, the SSs can only know of the success of their BW request transmission if they receive a response in the form of a $\mathrm{BW}$ grant in the subsequent frames. A station that does not receive a response to its $\mathrm{BW}$ request by a certain deadline (called T16, with a minimum length of $10 \mathrm{~ms}$ ) assumes that either a collision happened or resources are not available at the BS. In either case, since the SS cannot determine the cause, it assumes that a collision happened and uses an exponential binary back-off procedure to resolve the collision. The collision resolution and avoidance regulation of $802.16 \mathrm{MAC}$ requires each SS to wait a random number of TOs before attempting a transmission in the contention period. This number is called the random back-off number and is chosen from an interval of $(0, C W-$ 1 ), in which $C W$ is the contention window size and is initially set to the minimum or initial contention window size of $W$. After each collision (when

The response to the request is not received for T16 duration), the contention window size doubles $(C W=2 i \cdot W$ after $i$ th collision). The doubling continues until the maximum contention window size is reached after $m$ retransmissions $(C W=2 m \cdot W)$. The values of minimum and maximum contention window sizes can be dynamically adjusted by the BS. When a successful transmission happens or the packet is dropped due to reaching the retransmission limit, the contention window size is reset to $W$.

The size of the contention period is determined by the $\mathrm{BS}$ and may be different in each frame. If the random value of the back-off counter does not reach zero within a contention period, its countdown is frozen at the end of the contention period and resumes in the next contention period.

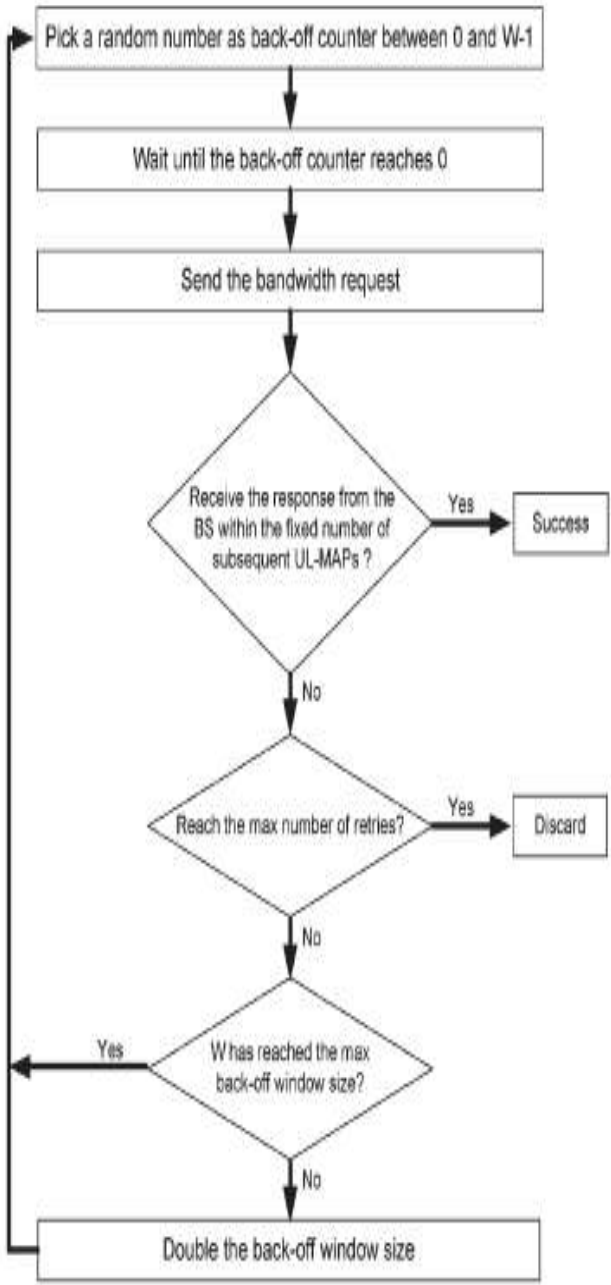

Fig. 2 Operation of Contention Resolution

\section{RELATED WORK}

In recent years, many researchers are interesting in IEEE 802.16 QoS research. There are many articles on the WiMAX QoS scheduling that have presented architectures and scheduling disciplines to guarantee QoS.

In [8][9] the introduction of Wimax is described. Broadband Wireless Access (BWA) has become the easiest way for 
wireless communication and a solution to rapid requirement of internet connection for data, voice and video service.

In [10 J. Lin and H. Sirisena, focuses on the analysis of QoS in WiMAX networks. It includes the definition of various service flows and their applications defined by the IEEE 802.16 standard

In [11], K. Wongthavarawat and A. Ganz presents an approach based on a fully centralized scheduling (GP Clike) scheme, where a global QoS agent collects all the necessary information on traffic flows, and takes decisions on traffic admission, scheduling, and resource allocation. Based on the complete global knowledge of the system, the deterministic QoS levels can be guaranteed.

In[1] Yousry Salaheldin Abdel-Hamid,formulated the new concept for contention based bandwidth request mechanism by driving the idea of using a random variable radix for backoff window instead of increasing the backoff window exponentially as defined in BEB algorithm. But the work does not specify any method for random request generation.

[2] Young dea lee, Sung Jun Park and SeungJune Yi proposed a concept of transferring back off parameters by Base Station to SS i.e. there is no need of calculating the value of back off counter explicitly by SS as it is provided by BS. But in this method it is observed that if response packet containing backoff value parameter dropped or lost due to some reason than SS would enter into a long wait state.

In [3] Jianhua He, Kun Yang, Ken Guild, and Hsiao-Hwa Chen defined the bandwidth request mechanism using piggybacking procedure.

In [4] Yaser Pourmohammadi Fallah; proposed an analytical model for contention based bandwidth request mechanism in wimax networks. He formulated method for random generation of bandwidth request using Poisson process.

In a nut shell, all the paper survey work concluded that there is no proper technique or any method is specified till date which generates a proper random back off counter value in order to increase contention resolution efficiency and other QoS parameters.

\section{PROBLEM FORMULATION}

IEEE 802.16 specification has been developed keeping in mind the strict QoS requirements of various kinds of flows. However there is no specification for a contention scheduling algorithm to meet these requirements. Hence, a scheduling algorithm must be developed for IEEE 802.16 in such a way so that it can cater to the QoS requirements of the different classes of flows.

We propose a scheduling architecture with an aim at providing the delay and $t$ guarantees of the various QoS sensitive flows. We will evaluate the performance of our algorithm by running extensive simulations. Based on the results of the simulations we will study the effectiveness of our algorithm in catering to the QoS needs of different types flows.

\section{PROPOSED WORK}

In conventional backoff procedures, the SS shall double its back-off window size if the current contention window size is smaller than the maximum back-off window size. The SS selects a fresh random number from 0 to $W-1$, where $W$ indicates the new back-off window size and repeats the deferring procedure. The SS can attempt to transmit BRs until the maximum number of retries is reached.

In conventional method-After sending request to the BS stations keeps waiting for UL-MAP-RES reply message from BS in order to get connected. There may be some other reasons that $\mathrm{SS}$ is not acknowledged:

$$
\begin{array}{ll}
\text { i. } & \text { Collision } \\
\text { ii. } & \text { Packet Drop } \\
\text { iii. } & \text { Channel Failure }
\end{array}
$$

The main drawback of this scheme is bandwidth wastage. i.e SS keeps waiting while there is bandwidth availability is present at BS.

This problem can be sorted out if SS station is made to send request frequently to BS i.e. SS station sends request after random time units frequently which is proposed solution in this paper.

Since several SSs can access the channel simultaneously during each frame, their respective transmissions may collide. The mandatory contention resolution algorithm is TBEB based, with an initial backoff window and a maximum backoff window controlled by BS. Meanwhile, SS will set its initial backoff window equal to the request backoff start whenever it has information to send and has to enter into the contention resolution process. SS shall randomly select a number, which indicates the number of contention transmission opportunities that SS shall defer before the transmission of bandwidth request, within its backoff window. The backoff process proceeds whenever collisions happen.

Since SS randomly selects a backoff number, so all SS will always tend to choose a small value of this number that can further prone to collision. Therefore a proper method must be specified to generate this random number so that all SSs can get the opportunity to transmit data.

\subsection{Proposed Contention Based Bandwidth Request Scheme in Wimax}

In this section, we analyze the performance of the contention access mode of 802.16 in terms of its capacity to deliver BW requests from stations to the BS. The analysis in this section is different from the classic slotted ALOHA system analysis in that we model the exponential back-off procedure for the first transmission and retransmissions and do not assume an infinite number of stations. In addition, our analysis considers each station rather than the system as a whole on the average. For our analysis, we assume that there are $n$ stations with traffic of either type BE or type nrtPS that use contention access to request $\mathrm{BW}$.

In 802.16 , the requests are generated based on the decisions made by a vendor-defined algorithm, whereas in 802.11 , the channel access is attempted for each packet. The model presented in this paper is much more detailed than the models for 802.11 to capture the precise operation of the 802.16 MAC 
and incorporate its different acknowledgement timeout procedure.

In our model, we do not assume any specific algorithm for $\mathrm{BW}$ request generation. Instead, we assume that requests are generated at random intervals, emulating the general behaviour of user-defined algorithms for request generation. In particular, we assume a Inverse Transformation Method (ITM) to be a good representation of the request generation pattern. Using a Inverse Transformation process is generally reasonable because requests for BW are generated for several independent connections within a station and by user-defined algorithms. It is worth emphasizing that the volume of the generated $\mathrm{BW}$ requests is not directly correlated with the volume of the actual data, as BW requests are generated based on a vendor-selected algorithm. If the request generation rate is high, requests are generated back to back. We call this mode persistent request generation or the saturation mode. The case where requests are generated with larger intervals is called infrequent request generation (also called the nonsaturation case)

\subsection{Back off Mechanism (CW: Contention Window)}

To manage the backoff window, a Binary Exponential Backoff (BEB) mechanism is used with WiMax. Whenever a backoff occurs, the backoff time(backoff counter) is randomly chosen in the range $\left(0, \mathrm{~W}_{\mathrm{i}}-1\right)$ where $\mathrm{W}_{\mathrm{i}}$ is value of contentioin window at ith transmission. After each unsuccessful transmission, the backoff window size is doubled, up to a maximum value $2 \mathrm{~mW}_{\mathrm{i}}$, where $\mathrm{W}_{\mathrm{i}}$ equals to $\left(\mathrm{W}_{\max }+1\right)$ and $\mathrm{m}$ is a specified number of attempts. Once the backoff window size reaches $\mathrm{W}_{\max }$, it will stay at the value of $\mathrm{W}_{\max }$ until it is reset. The value of $\mathrm{W}_{\mathrm{i}}$ will be reset to $\mathrm{W}_{\min }$ after every successful transmission of a data frame or a RTS frame, or when a retry counter reaches its limit.

Since a station uses CW to control the backoff counter for data transfer, how to set CW will affect the performance of the QOS. To optimize the performance of the QOS, we consider the backoff procedure as a progress to search the optimal value of $\mathrm{CW}$.

To solve this problem, we propose a dynamic CW resetting scheme to let the backoff counter oscillate around the optimal value.

\section{The proposed scheme is described as follows:}

A. After a successful transmission, $\mathrm{W}$ is set to the value:

$$
\mathrm{W}_{\mathrm{i}}=\max \left[\mathrm{W}_{\mathrm{i}} / 2, \mathrm{CWmin}+1\right] .
$$

B. Whenever a transmission fails, $\mathrm{W}$ is set to the value:

$$
\mathrm{W}_{\mathrm{i}}=\min \left[2 \mathrm{~W}_{\mathrm{i}}, \mathrm{CWmax}+1\right] \text {. }
$$

\section{Inverse Transformation method:}

Inverse transform sampling (also known as inversion sampling, the inverse probability integral transform, the inverse transformation method, Smirnov transform, golden rule,[1] etc.) is a basic method for pseudo-random number sampling,

i.e. for generating sample numbers at random from any probability distribution given its cumulative distribution function (cdf).
The basic idea is to uniformly sample a number $u$ between 0 and 1 , interpreted as a probability, and then return the largest number $\mathrm{x}$ from the domain of the distribution $\mathrm{p}(\mathrm{X})$ such that

$\mathrm{p}(-$-linfty $<\mathrm{X}<\mathrm{x}) \backslash$ le $\mathrm{u}$. For example, imagine that $\mathrm{p}(\mathrm{X})$ is the standard normal distribution (i.e. with mean 0 , standard deviation 1). Then if we choose $u=0.5$, we would return 0 , because $50 \%$ of the probability of a normal distribution occurs in the region where $X \backslash$ le 0 . Similarly, if we choose $u=0.95$, we would return $1.95996 \ldots$; if we choose $u=0.99$, we would return $2.5758 \ldots$; if we choose $\mathrm{u}=0.999999$, we would return $4.891638 \ldots ;$ etc.

Essentially, we are randomly choosing a proportion of the area under the curve and returning the number in the domain such that exactly this proportion of the area occurs to the left of that number. Intuitively, we are unlikely to choose a number in the tails because there is very little area in them: We'd have to pick a number very close to 0 or 1 .

Computationally, this method involves

Computing the quantile function of the distribution - in other words, computing the cumulative distribution function (CDF) of the distribution (which maps a number in the domain to a probability between 0 and 1 ) and then inverting that function. This is the source of the term "inverse" or "inversion" in most of the names for this method. Note that for a discrete distribution, computing the CDF is not in general too difficult: We simply add up the individual probabilities for the various points of the distribution. For a continuous distribution, however, we need to integrate the probability density function (PDF) of the distribution, which is impossible to do analytically for most distributions (including the normal distribution). As a result, this method may be computationally inefficient for many distributions and other methods are preferred; however, it is a useful method for building more generally applicable samplers such as those based on rejection sampling.

For the normal distribution, the lack of an analytical expression for the corresponding quantile function means that other methods (e.g. the Box-Muller transform) may be preferred computationally. It is often the case that, even for simple distributions, the inverse transform sampling method can be improved on[2]: see, for example, the ziggurat algorithm and rejection sampling. On the other hand, it is possible to approximate the quantile function of the normal distribution extremely accurately using moderate-degree polynomials, and in fact the method of doing this is fast enough that inversion sampling is now the default method for sampling from a normal distribution in the statistical package $\mathrm{R}$.

It is important to note that there is no universal algorithm for BW request generation, and using a model such as the one assumed here is inevitable. A BW will be granted if the BW request message is received successfully (no collision) and there is enough BW available. In the case of a collision or unavailability of BW, the station has to wait for some timeout duration (T16 seconds) and then start contending for a BW request again. Without loss of generality, we assume that the timeout value is $M$ frames long. 


\section{Inverse transform method : Discrete random variables}

- Want to generate a discrete random variable $X$ with pmf

$$
\mathrm{P}\left(X=x_{i}\right)=p_{i}, \quad i=1, \ldots, m
$$

- Consider the following algonithm

1. Generate a random number U

2. Transform $U$ into $X$ as follows.

$$
X=x_{j} \quad \text { if } \quad \sum_{i=1}^{j-1} R_{i} \leq U<\sum_{i=1} p_{i}
$$

- Proof the algorithm works...

$$
\begin{aligned}
\mathrm{P}\left(X=x_{j}\right) & =\mathrm{P}\left(\sum_{i=1}^{j-1} p_{i} \leq U<\sum_{i=1}^{j} p_{i}\right) \\
& =\sum_{i=1}^{1} p_{i}-\sum_{i=1}^{j-1} p_{i}=p_{j}
\end{aligned}
$$

- Suppose $x_{1}<x_{2}<\ldots<x_{m}$ then the cuf of $X F_{X}$ is.

$$
F_{X}(x)= \begin{cases}0, & x<x_{1+} \\ \Sigma_{i=1}^{j} p_{i,} & x_{j} \leq x<x_{j+1, j} \leq m-1 \\ 1, & x \geq x_{m}\end{cases}
$$

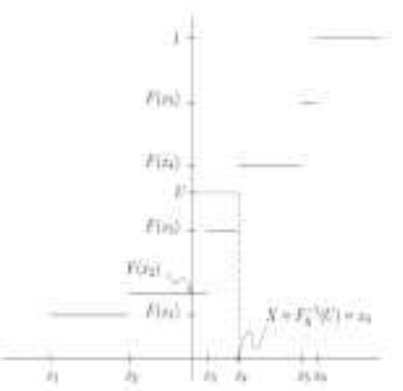

Thas ... $X=x_{j}$ if $F_{X}\left(x_{j-1}\right) \leq U<F_{X}\left(x_{j}\right)$

- If one defines the generalized inverse $F_{Y}^{-1}$ by

$$
F_{X}^{-1}(x)=\min \left\{y \mid F_{X}(y) \geq x\right\}
$$

then $\ldots=F_{X}^{-1}\left(C^{\prime}\right)$
Steps of algorithm

\section{Initialization: \\ Count $\leftarrow 0$}

For each packet generate

Check out bandwidth allocation:

If(true $)\{$

Process packet;

Count $=$ count +1 ;

Else\{

Send request randomly

(back off process start under contention period.

value of back off counter is generated randomly

using Inverse Transformation Method)

Count=count;

If(request acepted)

Occupy Bandwidth;

Count=count $+\mathbf{1}$;

Else\{

Send Request randomly;

Count=count;

\}

After developing contention resolution algorithm, we will compare the results with other latest developed Contention resolution algorithm and analyse the performance of proposed algorithm and thus extract the efficiency and scope of algorithm w. r. t. various QoS parameters.

\section{PERFORMANCE ANALYSIS}

Simulation is performed using OPNET Modeler 14.0. Initial assumptions are: Number of SSs $(\mathrm{N})=8$; no. Of $\mathrm{BS}=1$; number of retransmission limit $=8$; packet size 1024bits; bandwidth $=5 \mathrm{mbps}$; backoff window $\left(\mathrm{W}_{0}\right)$ size initially $=5$;

Following QoS parameters are considered while performing simulation-Delay, load and throughput in wimax. The modifications are made only to the contention resolution process and other operations are left as system default.

$\mathrm{X}$ will be the value of random back off counter in back off procedure generated by ITM. 
Council for Innovative Research

www.ijctonline.com ISSN: 2277-3061

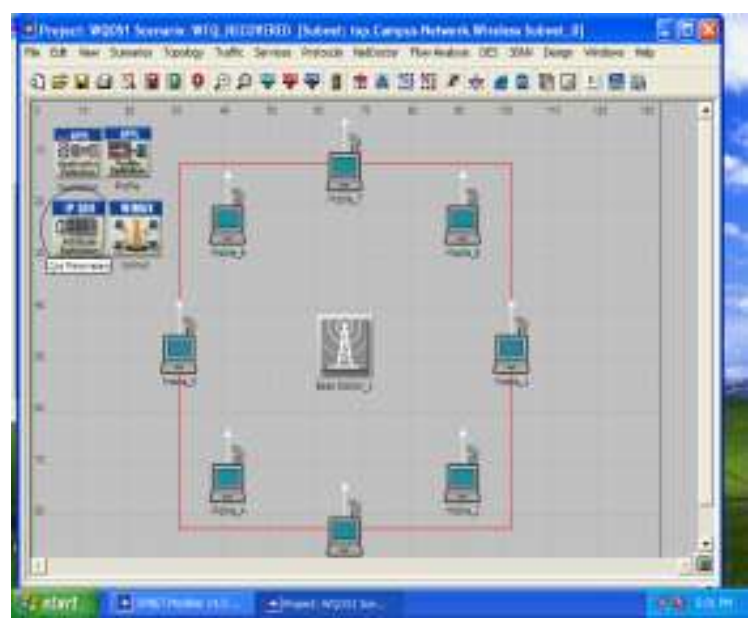

Fig.3 Simulation Scenario

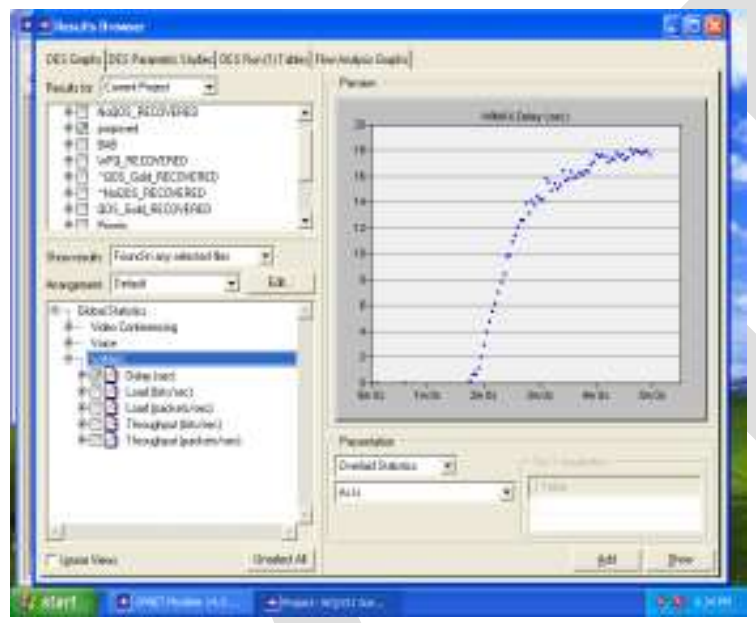

Fig .4 Packet delay / sec

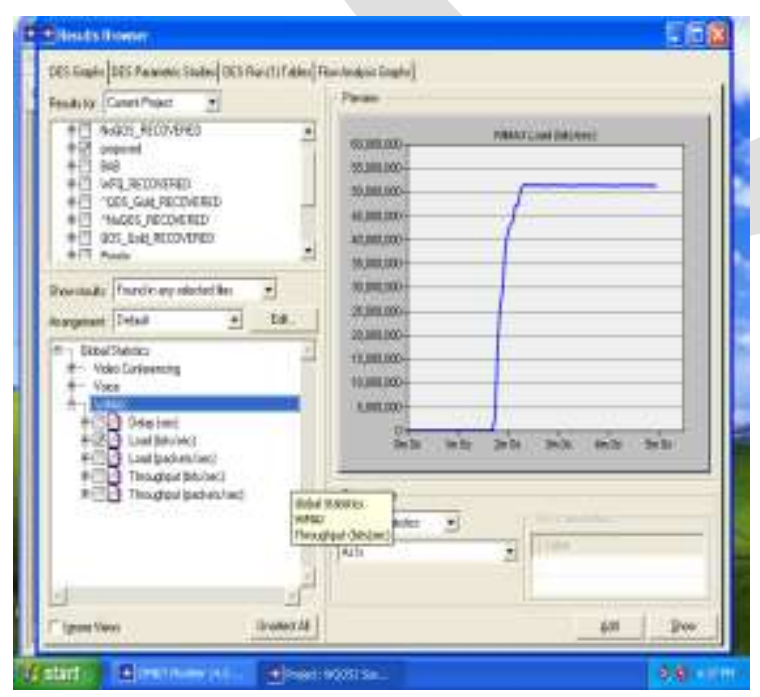

Fig5. Load- Bits/sec.
International Journal of Computers \& Technology

Volume 3, No. 1, AUG, 2012

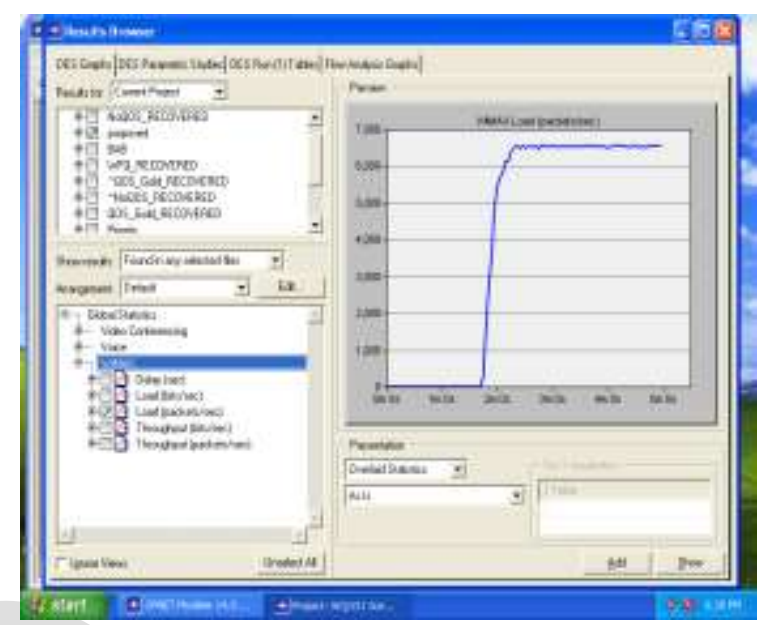

Fig. 6 Load packets / sec.

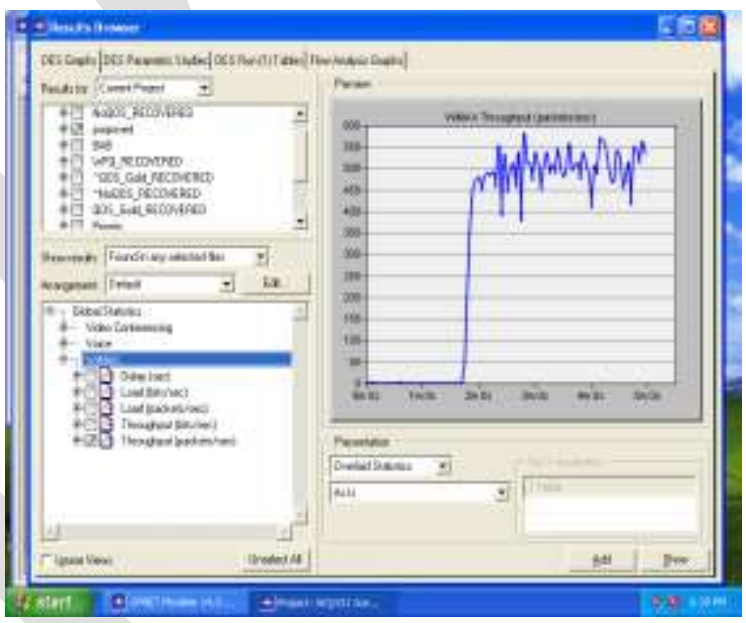

Fig.7 Throughput - Packets/sec.

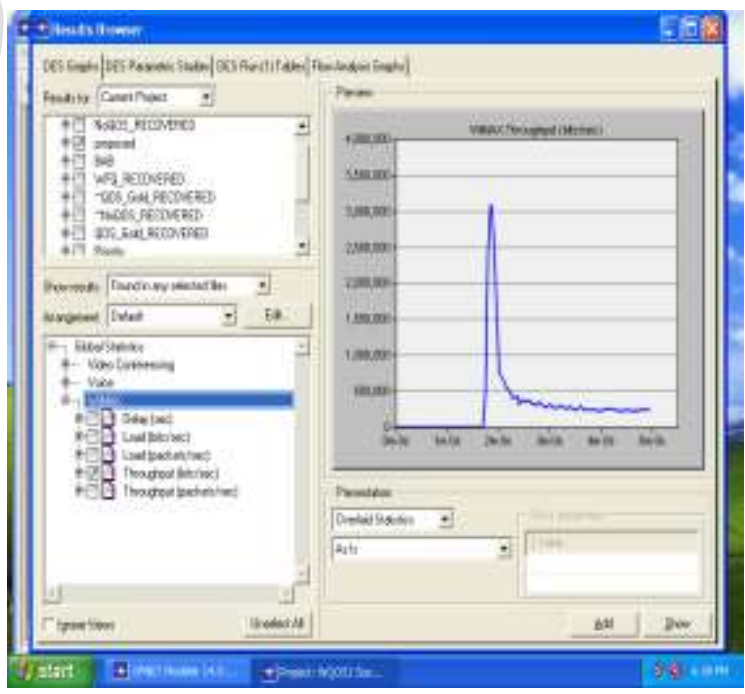

Fig. 8 Throughput- Bits/ sec. 


\section{CONCLUSION}

In this paper a new theory of Inverse Transformation Method has been considered in order to generate request randomly. In literature survey it has been observed that not much attention has been paid towards this area till date. After performing analysis we found that value of delay $(\mathrm{bits} / \mathrm{sec})=$ $71.6 \%$

$\operatorname{load}(\mathrm{bits} / \mathrm{sec})=61.4 \%$ load $(\mathrm{bits} / \mathrm{sec})=61.07$, throughput $(\mathrm{bits} / \mathrm{sec}$ )$=9.1 \%$, throughput $($ packets $/ \mathrm{sec})=53.9 \%$. With the proposed approach for WiMAX, delay can be reduced and throughput can be increased as compared to existing algorithms. Thus we conclude that in spite of various other techniques and algorithms implemented at different layers and levels, finding a new and optimal solution for bandwidth request regeneration randomly can also help in improving the QoS. In future there could be much more methods developed that can generate much optimum values for backoff counter values for retransmission.

\section{REFERENCE}

[1] On Markov Modeling of Random Access in Communication Systems; by Yousry Salaheldin Abdel-Hamid B.Sc. Ain Shams University, 1987 M.A.Sc. University of Victoria, 2003; c Yousry Salaheldin Abdel-Hamid,;University of Victoria, 2012

METHOD FOR SIGNALING BACKOFF INFORMATIION IN RANDOM ACCESS. Young dae lee, Sung Jun Park,Seun June Yi,Sun Duck Chuk;United State Patent Application Publication ;Publication No. US 2011/0216706 A1; pub date Sep. 082011

[3] On Bandwidth Request Mechanism with Piggyback in Fixed IEEE 802.16 Networks;Jianhua He, Kun Yang, Ken Guild, and Hsiao-Hwa Chen; IEEE TRANSACTIONS ON WIRELESS COMMUNICATIONS, VOL. 7, NO. 12, DECEMBER 2008

[4] Analytical Modeling of Contention-Based Bandwidth Request Mechanism in IEEE 802.16 Wireless Networks; Yaser Pourmohammadi Fallah, Member, IEEE, Farshid Agharebparast, Member, IEEE, Mahmood R. Minhas,Hussein M. Alnuweiri, Member, IEEE, and Victor C. M. Leung, Fellow, IEEE ; IEEE TRANSACTIONS ON VEHICULAR TECHNOLOGY, VOL. 57, NO. 5, SEPTEMBER 2008

[5] Waiting Queue Based Bandwidth Allocation Architecture for nrtPS and BE Services in IEEE 802.16 PMP Network; Wenfeng Du, Zhen Ji, Zhiqiang Wang, Weijia Jia; 978-1-4244-21084/08/\$25.00 @ 2008 IEEE

[6] A Comprehensive Analysis of Bandwidth Request Mechanisms in IEEE 802.;Networks;David Chuck, Kuan-Yu Chen, and J. Morris Chang, Senior Member, IEEE; IEEE TRANSACTIONS ON VEHICULAR TECHNOLOGY, VOL. 59, NO. 4, MAY 2010

[7] Base Station Assisted Backoff for Broadband Wireless AccessNetworks;Anbazhagan Rajesh and Rangaswamy Nakk; 2012 International Conference on System Engineering and Modeling (ICSEM 2012) IPCSIT vol. 34 (2012) (C) (2012) IACSIT Press, Singapore
[9] Roger B. Marks, "The IEEE 802.16 Wireless MAN Standard for Broadband Wireless Metropolitan Area Networks" IEEE Computer Society Distinguished Visitors Program, Jul. 2004.

[10] J. Lin and H. Sirisena, "Quality of service scheduling in IEEE 802.16 broadband wireless networks," in Proc. First International Conference on Industrial and Information Systems, Aug. 2006.

[11] K. Wongthavarawat and A. Ganz, "Packet scheduling for QoS support in IEEE 802.16 broadband wireless access systems," International Journal of Communication Systems, vol. 16, issue 1, pp. 81-96, Feb. 2003 